\title{
Oxaliplatin reverses the GLP-1R-mediated promotion of intrahepatic cholangiocarcinoma by altering FoxO1 signaling
}

\author{
BENDONG CHEN $^{1 *}$, WENYAN ZHOU ${ }^{2 *}$, WENCHAO ZHAO $^{3 *}$, PENG YUAN $^{1}$, CHAOFENG TANG $^{1}$, \\ GENWANG WANG ${ }^{1}$, JUNZHI LENG ${ }^{1}$, JINLONG MA ${ }^{4}$, XIAOWEN WANG ${ }^{4}$, YONGFENG HUI $^{1}$ and QI WANG ${ }^{1}$ \\ Departments of ${ }^{1}$ Hepatobiliary Surgery and ${ }^{2}$ Intensive Care Unit, The General Hospital of Ningxia Medical University, \\ Yinchuan, Ningxia Hui Autonomous Region $750004 ;{ }^{3}$ Department of Hepato-Biliary-Pancreatic Surgery, \\ Sixth Medical Center of People's Liberation Army General Hospital, Beijing 100043; \\ ${ }^{4}$ Department of Postgraduate, Ningxia Medical University, Yinchuan, Ningxia Hui Autonomous Region 750004, P.R. China
}

Received May 11, 2018; Accepted April 25, 2019

DOI: $10.3892 / 01.2019 .10497$

\begin{abstract}
Intrahepatic cholangiocarcinoma (ICC) is the second most common primary liver cancer, with a 5-year survival rate of $<10 \%$; effective drug treatment for ICC is currently lacking. Glucagon-like peptide-1 receptor (GLP-1R) is upregulated in ICC; however, the functions of GLP-1R in ICC remain unknown. In this study, the upregulation of GLP-1R was confirmed in ICC cells using reverse transcription-quantitative polymerase chain reaction and western blot analysis, and GLP-1R was determined to promote the migration and invasion of ICC cells using Transwell assays. This tumor-promoting effect depended on the upregulation of epithelial-mesenchymal transformation-associated proteins, which was mediated by the FoxO1 signaling pathway. It was also indicated that following oxaliplatin treatment, the effects of GLP-1R on EMT and invasion were reversed. This functional reversion was associated with the reduced phosphorylation of S256 in forkhead box O1 (FoxO1) and an increase in the levels of unphosphorylated FoxO1. These findings suggest that incretin-based therapies may increase the risk of ICC metastasis and should not be used solely for the treatment of patients with ICC.
\end{abstract}

\section{Introduction}

Intrahepatic cholangiocarcinoma (ICC) is a form of liver cancer typically diagnosed at advanced stages and with poor

Correspondence to: Dr Qi Wang, Department of Hepatobiliary Surgery, The General Hospital of Ningxia Medical University, 804 Shengli Street, Yinchuan, Ningxia Hui Autonomous Region 750004, P.R. China

E-mail:wq-6562@163.com

${ }^{*}$ Contributed equally

Key words: intrahepatic cholangiocarcinoma, glucagon-like peptide-1, forkhead box O1, epithelial-mesenchymal transformation, oxaliplatin prognosis; in the United States its incidence has increased to 3,000 cases annually (1). Despite the fact that surgery is the preferred treatment for early ICC, only $\sim 35 \%$ of patients have early stage disease that may be treated by surgical resection $(2,3)$. For patients with advanced stage or unresectable cholangiocarcinoma, the available systemic therapies are of limited effectiveness. The median overall survival time with the current standard of care chemotherapy regimen of gemcitabine and cisplatin is $<1$ year $(3,4)$. Therefore, the identification of novel antitumor targets for ICC is urgently required.

Glucagon-like peptide-1 (GLP-1) is an incretin hormone secreted by intestinal $\mathrm{L}$ cells in the distal intestine in response to nutrient ingestion $(5,6)$. Upon binding to the GLP-1 receptor (GLP-1R), GLP-1 affects blood glucose levels by stimulating insulin secretion, inhibiting glucagon secretion and gastric emptying, and reducing food intake (7-9). Due to its ability to regulate blood glucose levels, GLP-1 is now widely used in clinic for patients with diabetes (10). In addition to its hypoglycemic effect, GLP-1 alleviates inflammation in intraepithelial lymphocytes of intestinal mucosal epithelium, confers antioxidative and neurogenerative effects in the brain, and protects vascular functions in the cardiovascular system (11). GLP-1 and GLP-1R-associated signaling also promote tumor progression. Long-term GLP-1R activation was reported to increase the risk of pancreatic cancer development (12), and GLP-1R agonists were reported to promote neoplastic intestinal growth (13). In addition, GLP-1-based therapies have been used to treat thyroid carcinoma (14). It has been previously reported that GLP-1R protein expression is upregulated in ICC and that GLP-1R expression correlated with lymph node metastasis (15), indicating that GLP-1R is involved in ICC tumor progression. However, Exendin-4 with regards to diabetes, a GLP-1 analog that has similar functions as GLP-1, enhanced oxaliplatin-mediated tumor suppression in ICC (16). These conflicting data call for an in-depth study on the GLP-1R underlying mechanism of action in ICC.

Forkhead box O1 (FoxO1) is a member of the forkhead box family of transcription factors with a highly conserved DNA-binding domain (17). Although FOXO-mediated signal transduction pathways are evolutionarily conserved in most 
species, they seem to have been co-opted by differentiated tissues for a variety of specialized functions (18). For example, FoxO1 deficiency in $\mathrm{T}$ cells confers a survival defect and increased apoptosis, whereas in B cells, enforced expression of FoxO1 results in partial cell cycle arrest in addition to increased apoptosis (18). FoxO1 act as a tumor repressor while also maintaining cancer stem cells in some digestive malignancies including liver cancer, colorectal cancer, and gastric cancer (19). Some FoxO transcription factors promote antitumor activity by negatively regulating the expression of immunosuppressive proteins and by controlling the antitumor immune response, as well as the homeostasis and development of immune cells (20). It has been reported that GLP-1R signals activate FoxO1 in hepatocellular carcinoma (HCC) cells (21). However, it remains unclear whether GLP-1R regulates the phosphorylation of FoxO1 in other types of cancer. Thus, the present study aimed to determine whether GLP-1R regulates FoxO1 signaling and its function in ICC.

\section{Materials and methods}

Patients and specimens. ICC tumor specimens were obtained from patients who underwent surgical resection without preoperative treatment between April 2004 and May 2008 in the department of Hepatobiliary Surgery at the General Hospital of Ningxia Medical University (Yinchuan, China). All of the methods were approved by the Research Medical Ethics Committee of Ningxia Medical University (Yinchuan, China) and were carried out in accordance with the ethical guidelines of the Helsinki Declaration. Written informed consent was obtained from all patients.

Cell lines. The human ICC cell lines RBE and HCCC-9810, and the extrahepatic cholangiocarcinoma (ECC) cell lines QBC939 and SSP-25, were obtained from the Shanghai Cell Bank of the Chinese Academy of Sciences (Shanghai, China). Cells were cultured in DMEM (Sigma-Aldrich; Merck KGaA, Darmstadt, Germany) supplemented with $10 \%$ fetal bovine serum (FBS; Gibco; Thermo Fisher Scientific, Inc., Waltham, MA, USA) at $37^{\circ} \mathrm{C}$ with $5 \% \mathrm{CO}_{2}$. For oxaliplatin treatment, cells were cultured in normal culture media with $1 \mathrm{mg} / \mathrm{ml}$ oxaliplatin (Dalian Meilun Biotech Co., Ltd., ) at $37^{\circ} \mathrm{C}$ for $12 \mathrm{~h}$.

Western blot analysis. Briefly, HCC tissues or RBE, HCCC-9810, QBC939, SS_-25 cells were homogenized in SDS sample buffer (10\% Glycerol, $2 \%$ SDS, $0.01 \%$ bromophenol blue, $1.25 \%$ 2- $\beta$-mercaptoethanol, $25 \mathrm{mM}$ Tris- $\mathrm{HCl}, \mathrm{pH} 6.8$ ) using ULTRA-TURRAX ${ }^{\circledR}$ (IKA-Works, Inc., Wilmington, $\mathrm{NC}$, USA) at $4^{\circ} \mathrm{C}$. The protein concentration was determined with the Quick Start ${ }^{\mathrm{TM}}$ Bradford protein assay kit (Bio-Rad Laboratories, Inc., Hercules, CA, USA). Protein extracts $(10 \mu \mathrm{g})$ were loaded on $10 \%$ SDS-PAGE gels, and transferred to $0.45 \mu \mathrm{m}$ PVDF membranes (EMD Millipore, Billerica, MA, USA) using electro-blotting apparatus (Bio-Rad Laboratories, Inc.). After overnight blocking with 3\% BSA (Sigma-Aldrich; Merck $\mathrm{KGaA}$ ) at $4^{\circ} \mathrm{C}$, membranes were incubated with the following primary antibodies: GLP-1R (dilution, 1:1,000; cat. no. 26196-1-AP; Proteintech Group Inc., Chicago, IL, USA), FoxO1 (1:1,000; cat no. 18592-1-AP; Proteintech Group Inc.), phosphorylated (p)-FoxO1 (S256) (dilution, 1:1,000; cat. no. 9461; Cell Signaling Technology, Inc., Danvers, MA, USA), glyceraldehyde-3-phosphate dehydrogenase (GAPDH; dilution, 1:3,000; cat. no. 10494-1-AP; Proteintech Group Inc.) overnight at $4^{\circ} \mathrm{C}$, following with HRP-conjugated secondary antibody (dilution, 1:3,000; cat. no. 715-035-150; Jackson ImmunoResearch Laboratories, Inc., West Grove, PA, USA) at $37^{\circ} \mathrm{C}$ for $2 \mathrm{~h}$. Enhanced chemiluminescence was used for detection. The protein bands were quantified by using ImageJ software v1.42 (National Institutes of Health, Bethesda, MD, USA).

Reverse transcription-quantitative polymerase chain reaction $(R T-q P C R)$. Total RNA of all cell lines was extracted from cells using TRIzol ${ }^{\circledR}$ (Invitrogen; Thermo Fisher Scientific, Inc.), according to the manufacturer's protocols. The RNA was subsequently reverse transcribed at $95^{\circ} \mathrm{C}$ for $10 \mathrm{sec}$ followed by 40 cycles at $95^{\circ} \mathrm{C}$ for $5 \mathrm{sec}$ and at $60^{\circ} \mathrm{C}$ for $45 \mathrm{sec}$. The cDNA was used as the template for quantitative PCR using a Takara RNA PCR kit and SYBR Premix Ex Taq (Takara Bio Inc., Otsu, Japan) using Applied Biosystem's 7500 qPCR System (Applied Biosystems; Thermo Fisher Scientific, Inc.), according to the manufacturer's protocols. GAPDH was used as an internal control. The following primers were used: Human GLP-1R, sense 5'-GGTGCAGAAATGGCGAGAATA-3', anti-sense 5'-CCGGTTGCAGAACAAGTCTGT-3'; human FoxO1, sense 5'-GGATGTGCATTCTATGGTGTACC-3', anti-sense 5'-TTTCGGGATTGCTTATCTCAGAC-3'; human Cadherin 1 (CDH1), sense 5'-CCTAGATGAACCTTATGAGGCCA-3', anti-sense 5'-GCTGTAGAGGAGACGAGCATTAT-3'; human GAPDH, sense 5'-GAGTCAACGGATTTGGTCGT-3', anti-sense 5'-TTGATTTTGGAGGGATCTCG-3'; human CDH2, sense 5'-TGTATGTGGGCA AGATCCACT-3', anti-sense 5'-CTCGTCGATCAGGAAGATGGT-3'; human Snail family transcriptional repressor 1 (SNAI1), sense 5'-TCG GAAGCCTAACTACAGCGA-3', anti-sense 5'-AGATGAGCA TTGGCAGCGAG-3'; human SNAI2, sense 5'-CGAACT GGACACACATACAGTG-3', anti-sense 5'-CTGAGGATC TCTGGTTGTGGT-3'; human cyclin D2 (CCND2), sense 5'-ACCTTCCGCAGTGCTCCTA-3', anti-sense 5'-CCCAGC CAAGAAACGGTCC-3'; human cyclin-dependent kinase inhibitor 1A (CDKN1A), sense 5'-TGTCCGTCAGAACCC ATGC-3', anti-sense 5'-AAAGTCGAAGTTCCATCGCTC-3' ; Bcl2-interacting mediator (BIM), sense 5'-TGGAGCGGACAT GATAAGCAT-3', anti-sense 5'-AGCACAGGTGTCAACTAA ATCC-3'; NADPH oxidase activator (NOXA), sense 5'-TGC TACACAATGTGGCGTC-3', anti-sense 5'-ACTTGGACA TGGCCTCCCTA-3'; and superoxide dismutase 2 (SOD2), sense 5'-TTTCAATAAGGAACGGGGACAC-3', anti-sense 5'-GTGCTCCCACACATCAATCC-3'. The thermocycling conditions were as follows: $95^{\circ} \mathrm{C}$ for $2 \mathrm{~min}, 95^{\circ} \mathrm{C}$ for $5 \mathrm{sec}$, $60^{\circ} \mathrm{C}$ for $30 \mathrm{sec}, 72^{\circ} \mathrm{C}$ for $30 \mathrm{sec}, 40$ cycles and dissociation curve. All data were calculated using the $2^{-\Delta \Delta \mathrm{Cq}}$ method (22).

Immunohistochemistry (IHC). IHC was performed on formalin-fixed paraffin-embedded surgical specimens. In brief, tissue samples were fixed with $4 \%$ formalin overnight at $4^{\circ} \mathrm{C}$, followed by dehydration in $70,80,95$, and $100 \%$ ethanol. After paraffin embedding, the specimen was cut to 5 to $8 \mu \mathrm{m}$ thick sections. Tissue sections were dried at $60^{\circ} \mathrm{C}$ for $6 \mathrm{~h}$, dewaxed in xylene, rehydrated in a gradient concentration of 
ethyl alcohol (100, 75, 50 and 25\%) and endogenous peroxidase activity was blocked using $3 \%$ hydrogen peroxide at $37^{\circ} \mathrm{C}$ for $10 \mathrm{~min}$. Following antigen retrieval with citrate buffer in a microwave oven, tissue sections were incubated with GLP-1R (1:200 dilution), FoxO1 (1:100 dilution), or p-FoxO1 (1:100 dilution) primary antibodies at $4^{\circ} \mathrm{C}$ overnight. The tissue sections were treated with Primary Antibody Amplifier Quanto and HRP Polymer Quanto (Thermo Fisher Scientific, Inc.) prior to visualization with DAB solution (Thermo Fisher Scientific, Inc.) and counterstaining with hematoxylin (Thermo Fisher Scientific, Inc.) according to the manufacturer's protocols. Sections were visualized using a Nikon Eclipse Ti-s microscope (Nikon Corporation, Tokyo, Japan) at x20 magnification and analyzed using ImageJ software v1.42 (National Institutes of Health, Bethesda, MD, USA). Two independent pathologists of the Department of Pathology at the General Hospital of Ningxia Medical University (Yinchuan, China), who were blinded to the patients' clinicopathological data, provided the IHC staining scores. The extent of tumor cell staining in the tissues was graded as follows: $0,<5 ; 1,5-25 ; 2,26-50 ; 3$, 51-75 and 4 for $76-100 \%$ (21). The intensity of IHC staining was scored as follows: 0, no IHC signal; 1 , weak signal; 2 , moderate signal and 3 , strong signal. The final score used in the analysis was calculated by multiplying the extent score by the intensity score, and the final score ranged between 0-12. Values $\leq 6$ were considered low expression based on Receiver Operating Characteristic analysis. The images presented were selected randomly.

Small interfering RNA (siRNA) and plasmids. The siRNA (5'-GCGCTTCATCAAGCTGTTTAC-3') targeting GLP-1R was purchased from Sigma-Aldrich (Merck KGaA). A scrambled siRNA precursor (Scr) was used as the negative control. The siRNAs $(10 \mu \mathrm{g}$ for cells in $10 \mathrm{~cm}$ dish, $0.01 \mu \mathrm{g} / \mu \mathrm{l})$ were transfected into cells using Lipofectamine ${ }^{\circledR}$ 2000 (Thermo Fisher Scientific, Inc.), according to the manufacturer's protocols. Lentiviral vectors for the overexpression of GLP-1R were constructed by cloning target genes into pCDH-CMV-MCS-EF1-Puro (System Biosciences, Palo Alto, CA, USA; cat. no. CD510B-1) between the EcoRI and XbaI restriction sites; these vectors were also used for transfection (10 $\mu \mathrm{g}$ for cells in $10 \mathrm{~cm}$ dish, $0.01 \mu \mathrm{g} / \mu \mathrm{l}$ ). The FoxO1 S256D and FoxO1 S256A plasmids were constructed by site-directed mutagenesis. Point mutations of FOXO1 were generated by PCR-based mutagenesis using the GeneArt ${ }^{\circledR}$ Site-Directed Mutagenesis System (Invitrogen; Thermo Fisher Scientific, Inc.), according to the manufacturer's protocols. Briefly, mutagenesis PCR was performed in the reaction mixture, including wild-type (WT)-FoxO1, DNA methylase, Pfx DNA polymerase, and point-mutated primers. The PCR products were transformed into DH5 $\alpha-\mathrm{T} 1 \mathrm{E}$. coli competent cells (Tiangen Biotech, Co., Ltd., Beijing, China), and the positive colonies were analyzed by sequencing. Mutagenesis primer sequences for S256D were forward, 5'-AGGAGAAGAGCTGCAAGT ATGGACAACAACAGT-3' and reverse, 5'-ACTGTTGTT GTCCATACTTGCAGCTCTTCTCCT-3'. Primer sequences for S256A were forward, 5'-AGGAGAAGAGCTGCAGCA ATGGACAACAACAGT-3' and reverse, 5'-ACTGTTGTT GTCCATTGCTGCAGCTCTTCTCCT-3'. For all overexpression experiments, empty pCDH-CMV-MCS-EF1-Puro vector was used as the control, and transfection efficiency was assessed using qPCR and western blot analysis. Further experiments were performed $48 \mathrm{~h}$ after transfection.

Transwell assays. Transwell migration and invasion assays were performed in 12 -well Transwell plates $(8-\mu \mathrm{m}$ pore size), according to the manufacturer's protocols (Corning Incorporated, Corning, NY, USA). For invasion assays, the bottom of a Transwell chamber was coated with BD Matrigel Basement Membrane Matrix (BD Biosciences, San Jose, CA, USA). Cells $\left(1 \times 10^{5}\right)$ in basic culture medium without serum were added to the upper chamber, and the lower chamber was filled with culture medium containing 20\% FBS (Invitrogen; Thermo Fisher Scientific, Inc.) as a chemoattractant. Cell migration and invasion were determined after 24 and $48 \mathrm{~h}$, respectively. Cells on the upper side of the chamber were removed from the surface of the membrane by scrubbing, and cells on the lower surface of the membrane were fixed with $4 \%$ paraformaldehyde at room temperature for $10 \mathrm{~min}$ and stained with $0.1 \%$ crystal violet at room temperature for $10 \mathrm{~min}$. The numbers of cells were counted in five randomly selected microscopic fields for each filter using a Nikon Eclipse Ti-s microscope (Nikon Corporation, Tokyo, Japan) at $\times 20$ magnification.

Statistical analysis. All data were presented as mean \pm standard deviation. All statistical data were based on three separate repeated trials. Statistical analysis was performed with GraphPad Prism 5.0 software (GraphPad Software, Inc., La Jolla, CA, USA). Differences between two groups were examined by a Student's two-tailed t-test; multiple comparisons between the groups were performed using Student-Newman-Keuls method following one way analysis of variance. Correlations between two groups were analyzed using a nonparametric Spearman's R test. $\mathrm{P}<0.05$ was considered to indicate a statistically significant difference.

\section{Results}

GLP-1R promotes migration and invasion of ICC cells. It has been indicated previously that GLP-1R is upregulated in ICC tumor tissues (15). To investigate the role of GLP-1R in ICC cells, GLP-1R expression was measured in different cholangiocarcinoma cell lines by RT-qPCR and western blot analysis. It was indicated that mRNA and protein expression levels of GLP-1R were significantly higher in the ICC cell lines RBE and HCCC-9810 compared with the ECC lines QBC939 and SSP-25 (Fig. 1A and B). The expression of GLP-1R was subsequently knocked down in RBE and HCCC-9810 cells by RNA interference to determine the effects of GLP-1R expression on tumor cell migration and invasion. Knockdown of GLP-1R expression was confirmed by RT-qPCR and western blot analysis (Fig. 1C and D), and the Transwell assay demonstrated that RBE and HCCC-9810 tumor cells exhibited significantly reduced migration and invasion upon GLP-1R silencing (Fig. 1E and F). Furthermore, overexpression of GLP-1R significantly promoted ICC cell migration and invasion compared with the control (Fig. 1G-J). These data demonstrated that GLP-1R promotes tumor cell migration and invasion during ICC progression. 


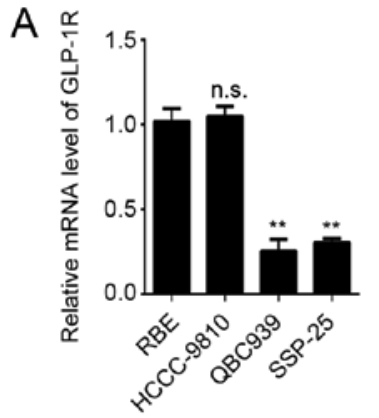

$E$

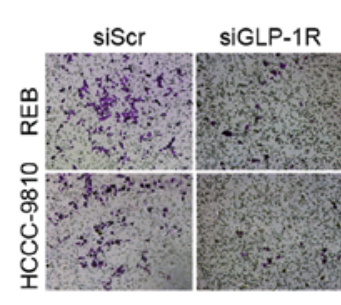

B
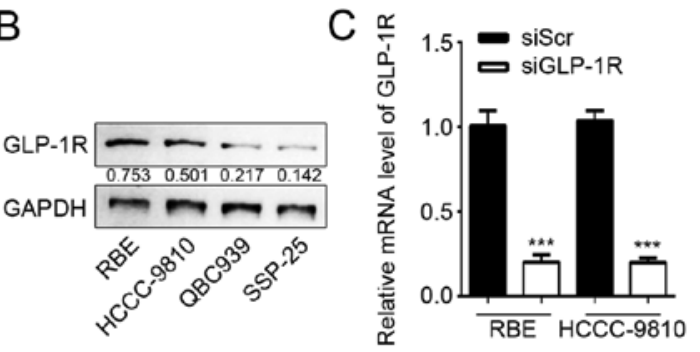

$\mathrm{F}$

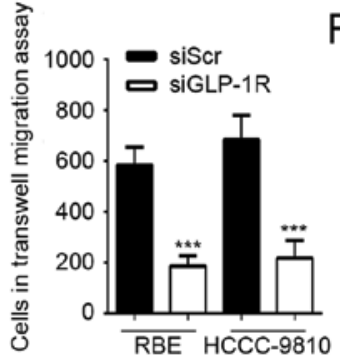

D

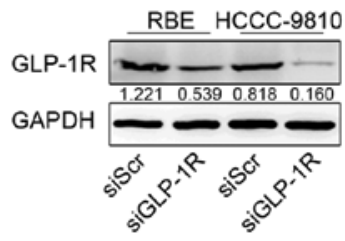

G

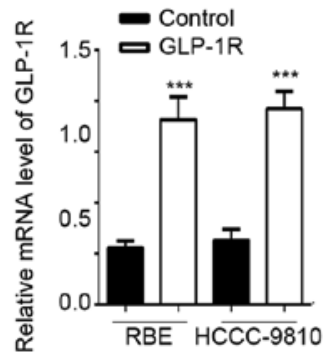

$\mathrm{H}$

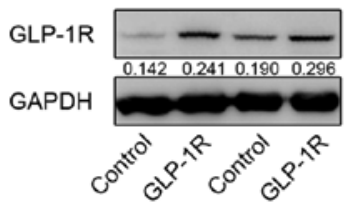

I
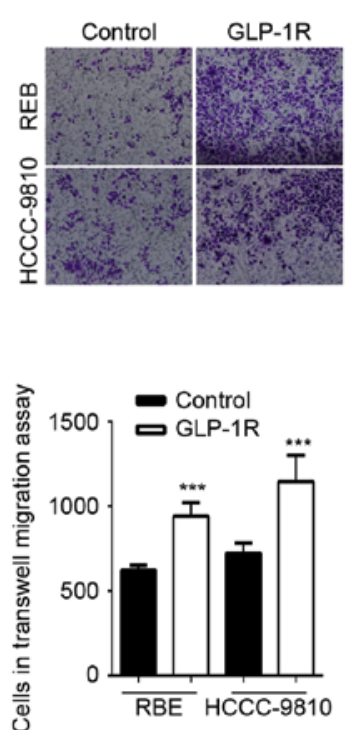
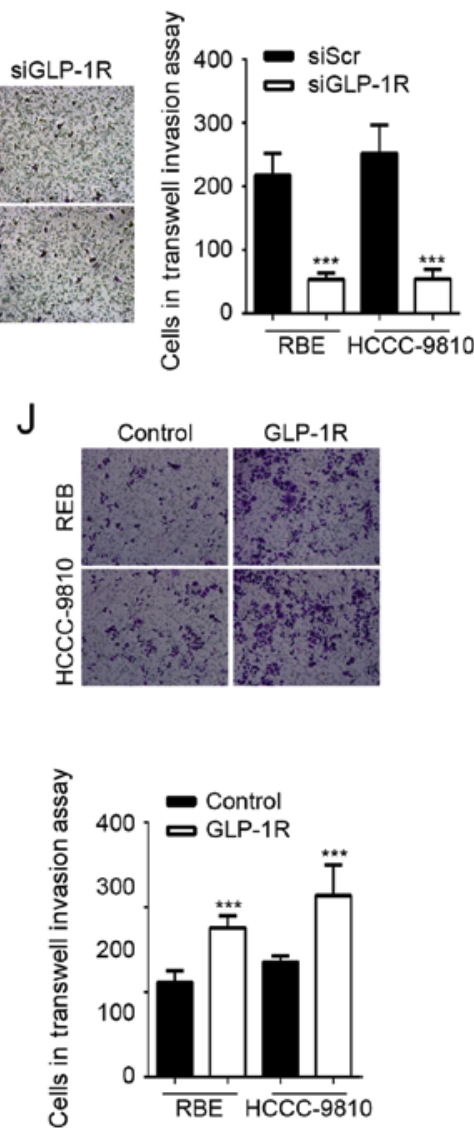

Figure 1. Knockdown of GLP-1R inhibits intrahepatic cholangiocarcinoma cell migration and invasion. (A) GLP-1R mRNA expression levels in different cholangiocarcinoma cell lines. ${ }^{* *} \mathrm{P}<0.01$ vs. RBE. (B) GLP-1R protein expression levels in different cholangiocarcinoma cell lines. Knockdown of GLP-1R in RBE and HCCC-9810 cells was confirmed by (C) western blot analysis and (D) reverse transcription-quantitative polymerase chain reaction. ${ }^{* * *} \mathrm{P}<0.01$ vs. SiScr. Transwell assays were used to determine the effects of GLP-1R silencing on the (E) migration and (F) invasion of RBE and HCCC-9810 cells. ${ }_{* * * *} \mathrm{P}<0.001$ vs. siScr. Overexpression of GLP-1R was confirmed by $(\mathrm{G})$ reverse transcription-quantitative polymerase chain reaction and $(\mathrm{H})$ western blot analysis. ${ }^{* * *} \mathrm{P}<0.001$ vs. Control. Transwell assays were used to determine the effect of GLP-1R overexpression on the (I) migration and (J) invasion of RBE and HCCC-9810 cells. ${ }^{* * *} \mathrm{P}<0.001$ vs. Control. n.s., not significant; GLP-1R; glucagon-like peptide-1 receptor; si, small interfering RNA. Representative images for Transwell assays were obtained using x20 magnification. n.s., not significant.

GP-1R functions in ICC by regulating FoxOl signaling. GLP-1R has been indicated to activate FoxO1 in obese mouse (21), and activated FoxO1 has been reported to be involved in maintaining cancer stem cells (19), benefiting tumor metastasis. Considering the effects of GLP-1R on tumor cell migration and invasion, it was speculated that GLP-1R may also modulate FoxO1 in ICC. The expression and phosphorylation state of FoxO1 under GLP-1R knockdown conditions was examined, and it was indicated that suppressed GLP-1R expression resulted in reduced phosphorylation of FoxO1 without affecting the FoxO1 mRNA and protein levels in ICC (Fig. 2A and B). The expression of some FoxO1-regulating genes, including CCND2, CDKN1A, BIM, NOXA and
SOD2 were also examined using RT-qPCR under GLP-1R knockdown conditions (18). As expected, GLP-1R knockdown significantly suppressed the expression of genes regulated by FoxO1, compared with the control (Fig. 2C). To verify this result, a correlation analyses was performed to determine the association between GLP-1R and FoxO1 expression, and phosphorylated FoxO1 expression by IHC of 20 tumor tissues from patients with ICC. IHC analysis revealed that the levels of GLP-1R protein correlated with the phosphorylation levels of FoxO1 ( $\mathrm{R}=0.8167 ; \mathrm{P}=0.0012)$, but not with FoxO1 expression ( $\mathrm{R}=-0.1858 ; \mathrm{P}=0.5631$ ) (Fig. 2D). However, the immunohistochemical staining using this antibody was not recommended by the manufacturer. Thus, to confirm 

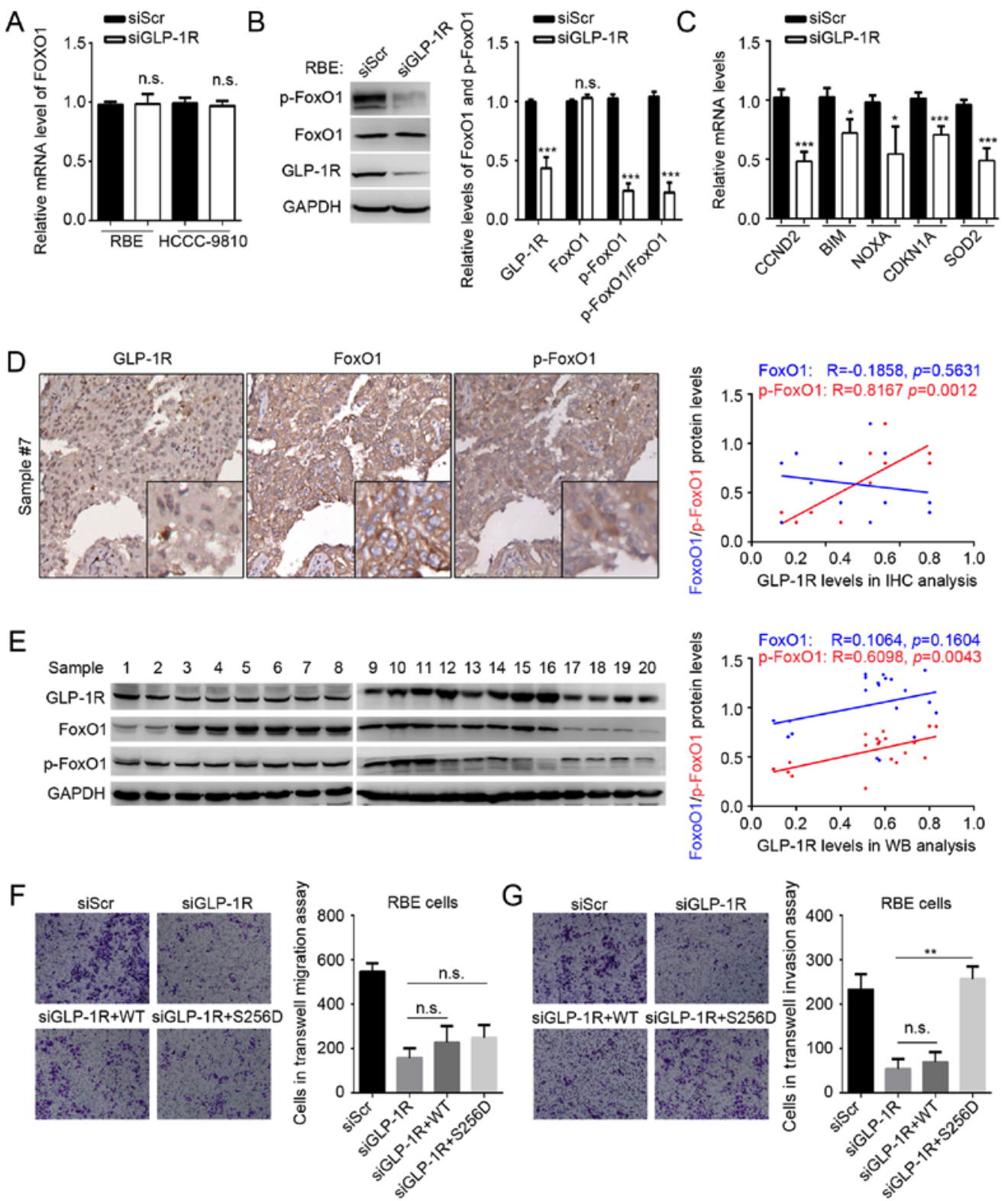

Figure 2. GLP-1R functions in ICC by regulating FoxO1 signaling. (A) The mRNA levels of FoxO1 in RBE and HCCC-9810 cells with GLP-1R knockdown were determined by reverse transcription-quantitative polymerase chain reaction analysis. (B) The protein level and the phosphorylation state of FoxO1 in RBE cells were determined by western blot analysis. The p-FoxO1/FoxO1 ratio was calculated as (p-protein/control)/(total protein/control). (C) Expression of genes targeted by FoxO1 were measured in RBE cells with GLP-1R knockdown. Correlations between GLP-1R levels, FoxO1 levels, and FoxO1 phosphorylation states were evaluated in 20 tumor tissues by (D) immunohistochemical and (E) western blot analyses. Transwell assays were used to determine the effects of FoxO1 and FoxO1S256D in the (F) migration and (G) invasion of RBE cells with GLP-1R knockdown. n.s., not significant; GLP-1R, glucagon-like peptide-1; FoxO1, forkhead box O1; p, phosphorylated; si, small interfering RNA. Representative images for Transwell assays were obtained at x20 magnification. n.s., not significant, ${ }^{*} \mathrm{P}<0.05$ vs. siGLP-1R, ${ }^{* *} \mathrm{P}<0.01$ vs. siGLP-1R or siGLP-1R+S256D, ${ }^{* * *} \mathrm{P}<0.001$ vs. siGLP-1R.

IHC results of the present study, western blot analysis was performed. As expected by using another 20 tissues for western blot analysis, GLP-1R protein expression correlated with the levels of phosphorylated FoxO1 ( $\mathrm{R}=0.6098 ; \mathrm{P}=0.0043)$, but not with FoxO1 expression ( $\mathrm{R}=0.1064 ; \mathrm{P}=0.1064)$ (Fig. 2E). Our previous findings indicated that upregulation of GLP-1R in ICC correlated with elevated lymph node metastasis (15); however, we failed to observe any significant correlation between IHC data and clinicopathological factors, which may be due to the small sample size $(n=20)$ in the current study. The aforementioned data suggested that GLP-1R regulates FoxO1 activation. In addition, a mutation (S256D) was constructed in FoxO1 that affects the phosphorylation of FoxO1. The mutation significantly increased the number of invasive cells compared with GLP-1R silencing, but not the abundance of migrating ICC cells under GLP-1R knockdown conditions (Fig. 2F and G). This indicated that GLP-1R functions in ICC by modulating the phosphorylation of FoxO1.

Oxaliplatin reverses the effects of GLP-1R on ICC invasion. Previously, it has been reported that during oxaliplatin treatment, an agonist of GLP-1R functions in ICC tumor suppression (16), and it has been indicated that the functions of tumor suppressors and oncogenic proteins are reversed during chemotherapy. For example, the effects of p53-mediated senescence on tumor cells could be reversed 
A
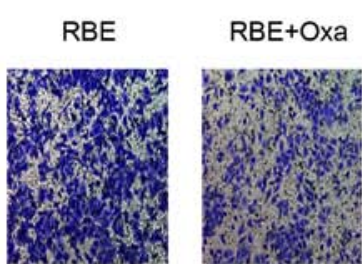

B

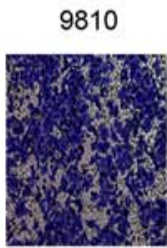

$9810+0 \times a$

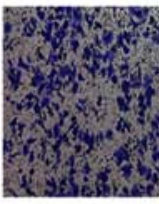

C
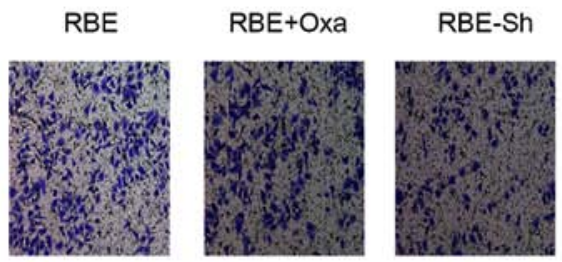

D

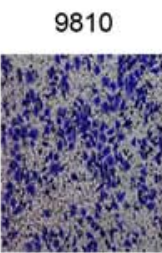

$9810+$ Oxa

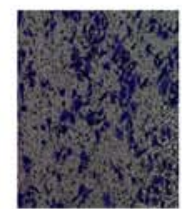

E

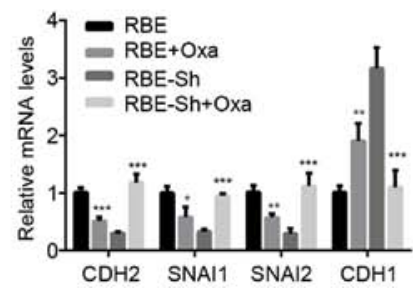

9810-Sh
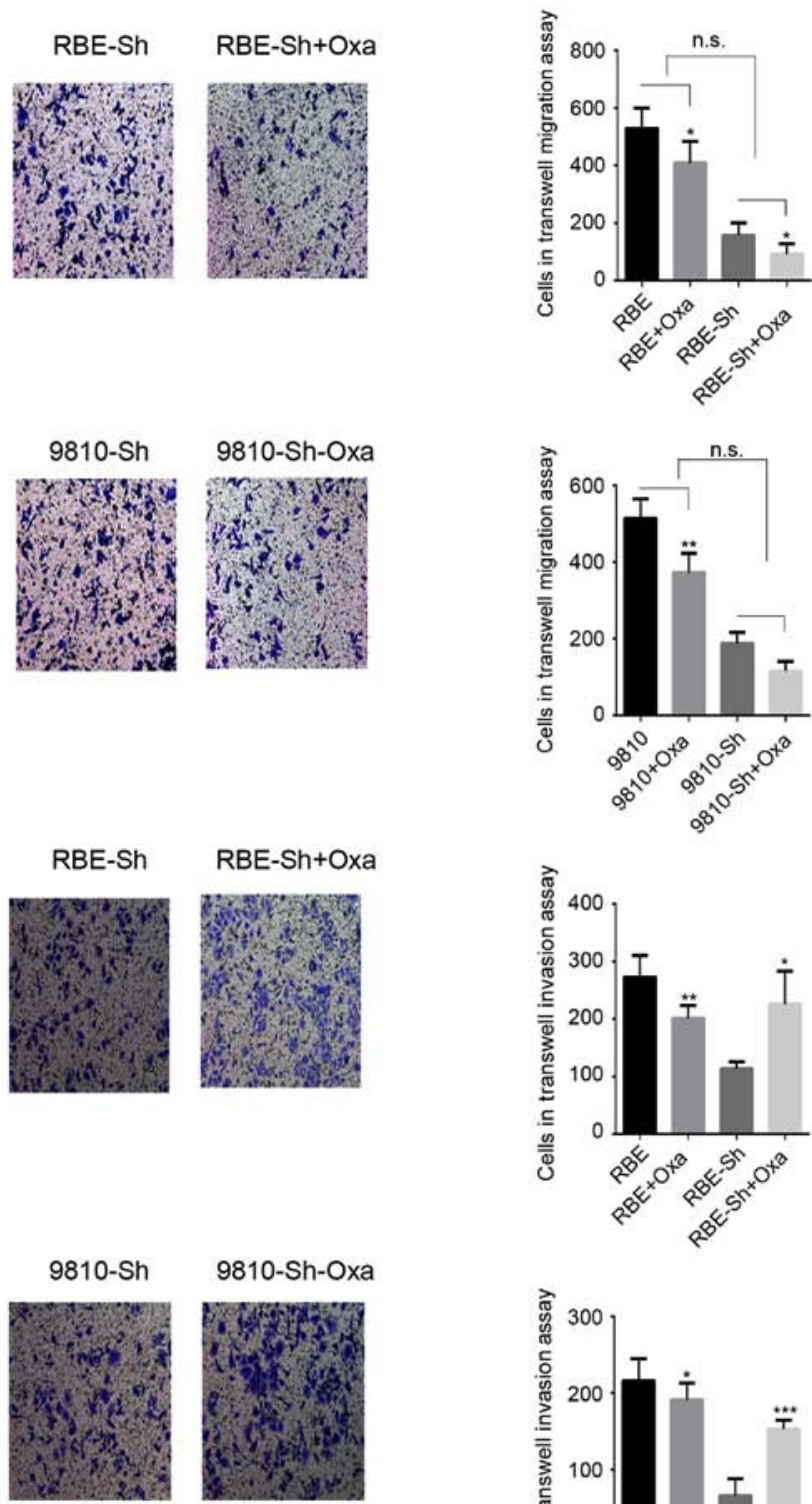

$\mathrm{F}$

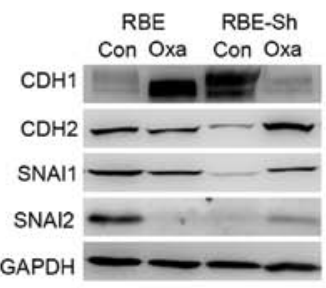

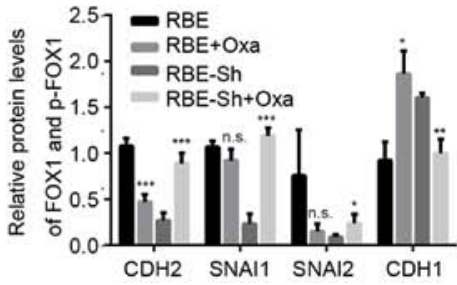

Figure 3. Effects of GLP-1R on intrahepatic cholangiocarcinoma invasion are reversed with oxaliplatin. (A-D) Transwell assays were used to determine the effects of GLP-1R silencing on migration and invasion of RBE and HCCC-9810 cells with OXA treatment. (E and F) The mRNA and protein levels of CDH2, SNAI1, SNAI2 and CDH1 in RBE cells with the indicated treatments. CDH1, E-cadherin; CHD2, N-cadherin; GLP-1R, glucagon-like peptide-1; OXA, oxaliplatin; si, small interfering RNA; n.s., not significant. ${ }^{*} \mathrm{P}<0.05$ vs. oxaliplatin treated, ${ }^{* *} \mathrm{P}<0.01$ vs. oxaliplatin treated, ${ }^{* * * *} \mathrm{P}<0.001$ vs. oxaliplatin treated.

under chemotherapy (23-25). It was speculated that chemotherapy may also alter the functions of GLP-1R in ICC. In the present study, oxaliplatin treatment significantly reduced the migration of tumor cells with or without GLP-1R-silencing. Also, GLP-1R knockdown did not suppress the inhibitory effects of oxaliplatin treatment on the migration of RBE and HCCC-9810 cells, indicating that GLP-1R may not be involved in the oxaliplatin-induced inhibition of migration (Fig. 3A and B). In addition, the inhibition of invasion by the GLP-1R knockdown was reversed with oxaliplatin treatment (Fig. 3C and D). Due to the fact that tumor invasion is associated with epithelial-mesenchymal transformation (EMT), the 
A
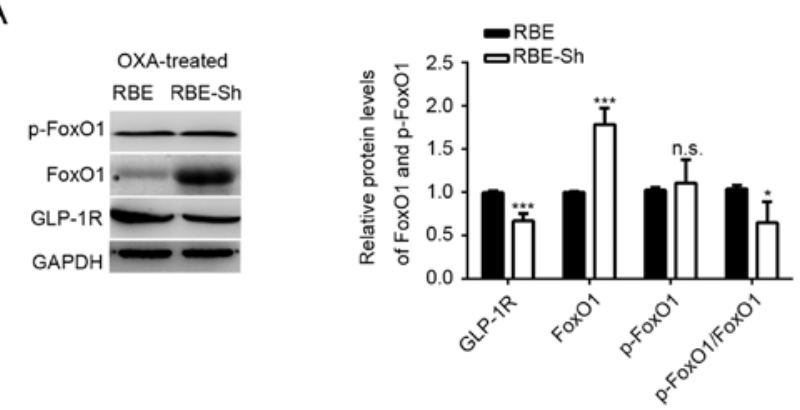

B

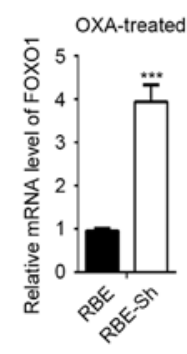

C

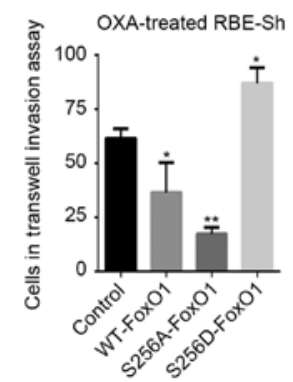

D

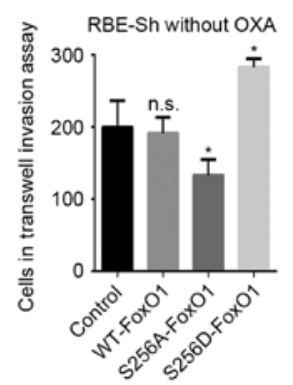

Figure 4. Oxaliplatin treatment suppresses GLP-1R-induced FoxO1 signaling. (A) The protein levels and the phosphorylation states of FoxO1 in oxaliplatin-treated REB cells. The p-FoxO1/FoxO1 ratio was calculated as (p-protein/control)/(total protein/control). ${ }^{*} \mathrm{P}<0.05$, ${ }^{* * *} \mathrm{P}<0.001$ vs. RBE. (B) The mRNA levels of FoxO1 in oxaliplatin-treated RBE cells with GLP-1R knockdown. ${ }^{* * *} \mathrm{P}<0.001$ vs. RBE. (C) Transwell assays were used to determine the effects of FoxO1 S256A and FoxO1 S256D on the invasion of oxaliplatin-treated RBE cells with GLP-1R knockdown. ${ }^{*} \mathrm{P}<0.05$, ${ }^{* *} \mathrm{P}<0.05$ vs. Control. (D) Transwell assays were used to determine the effects of FoxO1 S256A and FoxO1 S256D on the invasion of RBE cells treated without oxaliplatin. *P<0.05 vs. Control. GLP-1R, glucagon-like peptide-1; FoxO1, forkhead box O1; p, phosphorylated; si, small interfering RNA; n.s., not significant.

present study examined whether GLP-1R could regulate the expression of EMT-associated factors, such as CDH1, CDH2, SNAI1, and SNAI2, and whether oxaliplatin treatment could reverse these regulatory effects in ICC. The data showed that when comparing the RBE and RBE-sh groups, GLP-1R knockdown in RBE cells significantly decreased the mRNA levels of CDH2, SNAIl and SNAI2, and upregulated CDH1 (Fig. 3E), while these effects were reversed upon oxaliplatin treatment (Fig. 3E). Western blot analysis indicated similar results for the protein levels of EMT-associated factors (Fig. 3F). FoxO1 has been reported to regulate tumor cell growth; however, the present study did not investigate the effects of FOXO1 and its phosphorylation on ICC cell proliferation. The present study only suppressed the proliferation of ICC cells by using serum-free medium in the Transwell assays. Taking into consideration that changes in tumor growth may also influence the metastatic properties of ICC cells, further investigation is required to determine whether the effects of p-FoxO1 on metastasis depend on its regulation of tumor growth.

Oxaliplatin treatment reduces GLP-1R-induced FoxO1 signaling. The present study investigated whether FoxO1 signaling is involved in oxaliplatin-mediated changes in GLP-1R function. In contrast to the results of the GLP-1R knockdown without oxaliplatin treatment (Fig. 2B), the mRNA and protein levels of FoxO1 were significantly increased compared with the control, and the phosphorylation state of FoxO1 was notably unaffected in the GLP-1R knockdown RBE cells with oxaliplatin treatment (Fig. 4A and B). In addition, the FoxO1 S256D mutant with oxaliplatin treatment promoted the invasion of ICC cells, whereas overexpression of WT FoxO1 with oxaliplatin significantly inhibited invasion compared with the control (Fig. 4C), suggesting that an increase in unphosphorylated FoxO1 may be the key factor in reversing the effects of GLP-1R on invasion. An additional phosphorylation-deficient FoxO1 mutant, FoxO1 S256A, was created. The present study reported that, compared with the corresponding controls, the S256A mutation suppressed the invasion of ICC cells with or without oxaliplatin (Fig. 4D).

\section{Discussion}

Despite the fact that chemotherapy is a well-established cancer treatment, drug resistance has been observed for classical cytotoxic drugs and drugs that target specific molecules (26). Analyses of the associations between gene-expression profiles of tumor samples and the clinical responses of patients have revealed a strong correlation between EMT-associated gene expression and drug resistance (26). EMT is the process in which epithelial cells lose their apical-basal polarity and cell-cell adhesion properties, and transition to invasive mesenchymal cells (27). Cells undergoing EMT displayed reduced expression of epithelial genes, including CDH1 (also known as E-cadherin), increased expression of mesenchymal genes, including $\mathrm{CDH} 2$ (also known as $\mathrm{N}$-cadherin), and upregulated expression of a number of regulators of EMT, including SNAIL factors (SNAI1, also known as Snail, and SNAI2, also known as Slug) and FOX proteins (27-30). A number of these transcription factors, including FOXC2 and FOXM1, have also been reported to promote drug resistance $(31,32)$. FOXC2 was reported to increase the expression of ATP-binding cassette transporters, which enhance drug efflux, thereby promoting drug resistance (33). However, various members of the FOX transcription factor superfamily have the opposite effect on 
EMT-associated drug resistance. For example, FOXF2 has been determined to suppress FOXC2-mediated EMT (34). In the present study, it was demonstrated that the FOX transcription factor FoxO1, may inhibit EMT, but promoted this process in oxaliplatin-treated ICC cells. In addition, it was proposed that the phosphorylation of $\mathrm{S} 256$ in FoxO1 may be responsible for altering the function of FoxO1 during drug treatment.

FoxO1 modulates numerous target genes, including genes involved in apoptosis, cell cycle arrest and immune regulation, which suggests that FoxO1 would inhibit cell proliferation in cancer (35). Upregulation of FoxO1 has been reported to inhibit invasion and metastasis by reversing EMT in HCC (36). In this study, upregulation of FoxO1 in oxaliplatin-treated ICC cells was observed, and the data suggest that unphosphorylated FoxO1 may reverse EMT-mediated invasion. Previous studies have reported that the effects of FoxO1 depend on its activation, which can be influenced by its abundance, posttranscriptional modification, nuclear-cytoplasmic shuttling and subcellular localization (35-38). Among these activating factors, phosphorylation, particularly that of S256, is critical for FoxO1 function (37). Since FoxO1 S256D and S256A mutations conferred opposing effects on ICC invasion, we suggest that the phosphorylation of S256 may inhibit or reverse the function of FoxO1 in ICC. The phosphorylation of S256 may attenuate FoxO1 function by regulating its degradation (37-39); however, increased phosphorylation of S256 in oxaliplatin-treated ICC cells did not indicate reduced FoxO1 expression levels, and no significant correlation between the FoxO1 level and its phosphorylation in ICC tissues was reported. These data suggest that phosphorylation of S256 affects the function of FoxO1 in ICC via other mechanisms, which will be investigated in future studies.

It has been reported that exendin- 4 enhances the phosphorylation of FoxO1 in liver and HCC cells (21). Geniposide, an agonist of GLP-1R, also increased the levels of cytosolic phosphorylated FoxO1 in pancreatic $\beta$-cells and primary cortical neurons to inhibit apoptosis $(40,41)$. Our data confirms the effects of GLP-1R on the FoxO1 signaling pathway. In addition, GLP-1R has been reported to regulate the nuclear translocation of activated FoxO1 (42). Since phosphorylation of S256 may modulate the transactivation and nuclear targeting of FoxO1 (37-39), it appears that GLP-1R functions in ICC by regulating the phosphorylation of S256 in FoxO1. In addition, despite that GLP-1-induced FoxO1 activation promotes proliferation in early stage pancreatitis (42), FoxO1 signaling reversed the effects of GLP-1 on proliferation in pancreatic cancer (43), indicating that GLP-1-induced FoxO1 activation serves different roles under different conditions. Our data is in accordance with this conclusion and suggests that the effects of GLP-1R-associated therapy may vary under different conditions.

Incretin therapy, which involves GLP-1 receptor agonists, has recently become more popular among patients with tumors located in the digestive system (9). Incretin therapy provides effective glycemic control with a low risk of hypoglycemia, as well as improvements in lipid profiles, weight loss and insulin resistance (44). However, GLP-1R activation directly enhances proliferation and promotes cell survival in a number of tissues and cells, including B cells, cardiomyocytes, fibroblasts and neurons (45). In accordance with these oncogenic effects, our data revealed that upregulation of GLP-1R promoted EMT and enhanced tumor invasion. Nevertheless, GLP-1R functions as a tumor suppressor in endometrial cancer and pancreatic cancers $(46,47)$. Therefore, GLP-1R serves different roles in different types of cancer, and further research should focus on the underlying mechanisms of GLP-1R in specific cancers. Since the function of GLP-1R was reversed during oxaliplatin treatment, we suggest that agonists of GLP-1R have potential as chemotherapy boosters. For patients that have received surgical or other treatments, administration of these agonists requires further verification. In addition, although gemcitabine and cisplatin are commonly used to treat those with inoperable ICC, patients respond poorly to these chemotherapies (48). Future studies should focus on combinatorial treatments, including gemcitabine and cisplatin or gemcitabine, cisplatin, and oxaliplatin. In addition, oxaliplatin suppressed EMT in ICC cells and GLP-1R knockdown also suppressed EMT in ICC cells. However, oxaliplatin partially reversed GLP-1R knockdown-induced cell invasion. The potential mechanism of GLP-1R requires further research.

In conclusion, this study indicated that GLP-1R promotes ICC cell migration and invasion in vitro, and this tumor-promoting effect depended on the upregulation of EMT-associated proteins and was mediated by the FoxO1 signaling pathway. The effects of GLP-1R on EMT and invasion were reversed with oxaliplatin, which was associated with reduced phosphorylation of S256 in FoxO1 and an increase in unphosphorylated FoxO1. Our data also suggest that incretin-based therapies may increase the risk of ICC metastasis; thus, such treatments should not be solely used to treat patients with ICC. Additionally, GLP-1R agonists could be used as boosters for certain types of chemotherapies.

The present study indicated GLP-1R is upregulated in ICC, and incretin-based therapies may increase the risk of ICC metastasis. Aberrant upregulation of GLP-1R promotes ICC cell migration and invasion. Therefore, it should not be used solely for the treatment of patients with diabetes and ICC. In addition, oxaliplatin was proposed to reverse the incretin-mediated promotion of tumor invasion by altering FoxO1 signaling in ICC.

\section{Acknowledgements}

Not applicable.

\section{Funding}

The present study was funded by the Nature Science Foundation of Ningxia (grants no. NZ14132, NGY2016122, and XT201323).

\section{Availability of data and material}

All data generated or analyzed during this study are included in this published article.

\section{Authors' contributions}

BC, WYZ, WCZ, PY, CT, GW, JL, JM, XW, YH, and QW contributed to the study design, analysis, and interpretation of the data. BC conceived the study. WYZ, WCZ, PY, CT and GW 
performed the experiments. JL, JM, XW and YH performed the statistical analyses. BC drafted the manuscript. QW supervised the study and prepared the manuscript. All authors read and approved the final manuscript.

\section{Ethics approval and consent to participate}

The present study was approved by the Research Medical Ethics Committee of Ningxia Medical University (Yinchuan, China) and was carried out in accordance with the approved guidelines. Written informed consent was obtained from all of patients.

\section{Patient consent for publication}

Not applicable.

\section{Competing interests}

The authors declare that they have no competing interests.

\section{References}

1. Gatto $\mathrm{M}$ and Alvaro D: New insights on cholangiocarcinoma. World J Gastrointest Oncol 2: 136-145, 2010.

2. Jarnagin WR, Fong Y, DeMatteo RP, Gonen M, Burke EC, Bodniewicz BS J, Youssef BA M, Klimstra D and Blumgart LH: Staging, resectability, and outcome in 225 patients with hilar cholangiocarcinoma. Ann Surg 234: 507-519, 2001.

3. Rizvi S, Khan SA, Hallemeier CL, Kelley RK and Gores GJ: Cholangiocarcinoma-evolving concepts and therapeutic strategies. Nat Rev Clin Oncol 15: 95-111, 2018.

4. Valle J, Wasan H, Palmer DH, Cunningham D, Anthoney A, Maraveyas A, Madhusudan S, Iveson T, Hughes S, Pereira SP, et al: Cisplatin plus gemcitabine versus gemcitabine for biliary tract cancer. N Engl J Med 362: 1273-1281, 2010.

5. Tebay LE, Robertson H, Durant ST, Vitale SR, Penning TM, Dinkova-Kostova AT and Hayes JD: Mechanisms of activation of the transcription factor Nrf2 by redox stressors, nutrient cues, and energy status and the pathways through which it attenuates degenerative disease. Free Radic Biol Med 88: 108-146, 2015.

6. Orskov C, Rabenhøj L, Wettergren A, Kofod H and Holst JJ: Tissue and plasma concentrations of amidated and glycine-extended glucagon-like peptide I in humans. Diabetes 43: 535-539, 1994.

7. Lee YS and Jun HS: Anti-diabetic actions of glucagon-like peptide-1 on pancreatic beta-cells. Metabolism 63: 9-19, 2014.

8. Karaca M, Magnan C and Kargar C: Functional pancreatic beta-cell mass: Involvement in type 2 diabetes and therapeutic intervention. Diabetes Metab 35: 77-84, 2009.

9. Drucker DJ: The biology of incretin hormones. Cell Metab 3: 153-165, 2006.

10. Tremblay AJ, Lamarche B, Deacon CF, Weisnagel SJ and Couture P: Effects of sitagliptin therapy on markers of low-grade inflammation and cell adhesion molecules in patients with type 2 diabetes. Metabolism 63: 1141-1148, 2014.

11. Drucker DJ, Habener JF and Holst JJ: Discovery, characterization, and clinical development of the glucagon-like peptides. J Clin Invest 127: 4217-4227, 2017.

12. Elashoff M, Matveyenko AV, Gier B, Elashoff R and Butler PC: Pancreatitis, pancreatic, and thyroid cancer with glucagon-like peptide-1-based therapies. Gastroenterology 141: 150-156, 2011.

13. Koehler JA, Baggio LL, Yusta B, Longuet C, Rowland KJ, Cao X Holland D, Brubaker PL and Drucker DJ: GLP-1R agonists promote normal and neoplastic intestinal growth through mechanisms requiring Fgf7. Cell Metab 21: 379-391, 2015.

14. Spranger J, Gundert-Remy U and Stammschulte T: GLP-1-based therapies: The dilemma of uncertainty. Gastroenterology 141: 20-23, 2011.

15. Chen BD, Zhao WC, Dong JD and Sima H: Expression of GLP-1R protein and its clinical role in intrahepatic cholangiocarcinoma tissues. Mol Biol Rep 41: 4313-4320, 2014.
16. Chen BD, Zhao WC, Jia QA, Zhou WY, Bu Y, Wang ZZ, Wang F, Wu WJ and Wang Q: Effect of the GLP-1 analog exendin-4 and oxaliplatin on intrahepatic cholangiocarcinoma cell line and mouse model. Int J Mol Sci 14: 24293-24304, 2013.

17. Calnan DR and Brunet A: The FoxO code. Oncogene 27: 2276-2288, 2008.

18. Hedrick SM: The cunning little vixen: Foxo and the cycle of life and death. Nat Immunol 10: 1057-1063, 2009.

19. Lam EW, Brosens JJ, Gomes AR and Koo CY: Forkhead box proteins: Tuning forks for transcriptional harmony. Nat Rev Cancer 13: 482-495, 2013.

20. Deng Y, Wang F, Hughes T and Yu J: FOXOs in cancer immunity: Knowns and unknowns. Semin Cancer Biol 50: 53-64, 2018.

21. Lee J, Hong SW, Chae SW, Kim DH, Choi JH, Bae JC, Park SE, Rhee EJ, Park CY, Oh KW, et al: Exendin-4 improves steatohepatitis by increasing Sirtl expression in high-fat diet-induced obese C57BL/6J mice. PLoS One 7: e31394, 2012.

22. Livak KJ and Schmittgen TD: Analysis of relative gene expression data using real-time quantitative PCR and the 2(-Delta Delta C(T)) method. Methods 25: 402-408, 2001.

23. Jackson JG, Pant V, Li Q, Chang LL, Quintás-Cardama A, Garza D, Tavana O, Yang P, Manshouri T, Li Y, et al: p53-mediated senescence impairs the apoptotic response to chemotherapy and clinical outcome in breast cancer. Cancer Cell 21: 793-806, 2012.

24. Choi W,Porten S,KimS, Willis D,PlimackER,Hoffman-Censits J, Roth B, Cheng T, Tran M, Lee IL, et al: Identification of distinct basal and luminal subtypes of muscle-invasive bladder cancer with different sensitivities to frontline chemotherapy. Cancer Cell 25: 152-165, 2014.

25. Ihle NT, Byers LA, Kim ES, Saintigny P,Lee JJ, Blumenschein GR, Tsao A, Liu S, Larsen JE, Wang J, et al: Effect of KRAS oncogene substitutions on protein behavior: Implications for signaling and clinical outcome. J Natl Cancer Inst 104: 228-239, 2012.

26. Shibue T and Weinberg RA: EMT, CSCs, and drug resistance: The mechanistic link and clinical implications. Nat Rev Clin Oncol 14: 611-629, 2017.

27. Lamouille S, Xu J and Derynck R: Molecular mechanisms of epithelial-mesenchymal transition. Nat Rev Mol Cell Biol 15: 178-196, 2014.

28. Xia L, Huang W, Tian D, Zhu H, Qi X, Chen Z, Zhang Y, Hu H, Fan D, Nie Y and Wu K: Overexpression of forkhead box C1 promotes tumor metastasis and indicates poor prognosis in hepatocellular carcinoma. Hepatology 57: 610-624, 2013.

29. Li W, Zhang Z, Liu X, Cheng X, Zhang Y, Han X, Zhang Y, Liu S, Yang J, Xu B, et al: The FOXN3-NEAT1-SIN3A repressor complex promotes progression of hormonally responsive breast cancer. J Clin Invest 127: 3421-3440, 2017.

30. Kim T, Ha HI, Kim N, Yi O, Lee SH and Choi Y: Adrm1 interacts with Atp6v0d2 and regulates osteoclast differentiation. Biochem Biophys Res Commun 390: 585-590, 2009.

31. Zhou Z, Zhang L, Xie B, Wang X, Yang X, Ding N, Zhang J, Liu Q, Tan G, Feng D and Sun LQ: FOXC2 promotes chemoresistance in nasopharyngeal carcinomas via induction of epithelial mesenchymal transition. Cancer Lett 363: 137-145, 2015.

32. Chiu WT, Huang YF, Tsai HY, Chen CC, Chang CH, Huang SC, Hsu KF and Chou CY: FOXM1 confers to epithelial-mesenchymal transition, stemness and chemoresistance in epithelial ovarian carcinoma cells. Oncotarget 6: 2349-2365, 2015.

33. Saxena M, Stephens MA, Pathak $H$ and Rangarajan A: Transcription factors that mediate epithelial-mesenchymal transition lead to multidrug resistance by upregulating ABC transporters. Cell Death Dis 2: e179, 2011

34. Cai J, Tian AX, Wang QS, Kong PZ, Du X, Li XQ and Feng YM: FOXF2 suppresses the FOXC2-mediated epithelial-mesenchymal transition and multidrug resistance of basal-like breast cancer. Cancer Lett 367: 129-137, 2015.

35. Xing YQ, Li A, Yang Y, Li XX, Zhang LN and Guo HC: The regulation of FOXO1 and its role in disease progression. Life Sci 193: 124-131, 2018.

36. Dong T, Zhang Y, Chen Y, Liu P, An T, Zhang J, Yang H, Zhu W and Yang X: FOXO1 inhibits the invasion and metastasis of hepatocellular carcinoma by reversing ZEB2-induced epithelial-mesenchymal transition. Oncotarget 8: 1703-1713, 2017.

37. Zhang X, Gan L, Pan H, Guo S, He X, Olson ST, Mesecar A, Adam S and Unterman TG: Phosphorylation of serine 256 suppresses transactivation by FKHR (FOXO1) by multiple mechanisms. Direct and indirect effects on nuclear/cytoplasmic shuttling and DNA binding. J Biol Chem 277: 45276-45284, 2002 . 
38. Huang H, Regan KM, Wang F, Wang D, Smith DI, van Deursen JM and Tindall DJ: Skp2 inhibits FOXO1 in tumor suppression through ubiquitin-mediated degradation. Proc Natl Acad Sci USA 102: 1649-1654, 2005.

39. Rena G, Prescott AR, Guo S, Cohen P and Unterman TG: Roles of the forkhead in rhabdomyosarcoma (FKHR) phosphorylation sites in regulating 14-3-3 binding, transactivation and nuclear targetting. Biochem J 354: 605-612, 2001.

40. Liu J, Yin F, Xiao H, Guo L and Gao X: Glucagon-like peptide 1 receptor plays an essential role in geniposide attenuating lipotoxicity-induced $\beta$-cell apoptosis. Toxicol In Vitro 26: 1093-1097, 2012.

41. Zhang Y, Xia Z, Liu J and Yin F: Cell signaling mechanisms by which geniposide regulates insulin-degrading enzyme expression in primary cortical neurons. CNS Neurol Disord Drug Targets 14: 370-377, 2015.

42. Wewer Albrechtsen NJ, Albrechtsen R, Bremholm L, Svendsen B, Kuhre RE, Poulsen SS, Christiansen CB, Jensen EP, Janus C, Hilsted L, et al: Glucagon-like peptide 1 receptor signaling in acinar cells causes growth-dependent release of pancreatic enzymes. Cell Rep 17: 2845-2856, 2016.

43. Roy SK, Chen Q, Fu J, Shankar S and Srivastava RK: Resveratrol inhibits growth of orthotopic pancreatic tumors through activation of FOXO transcription factors. PLoS One 6: e25166, 2011.
44. Forst T, Weber MM and Pfutzner A: Cardiovascular benefits of GLP-1-based herapies in patients with diabetes mellitus type 2: Effects on endothelial and vascular dysfunction beyond glycemic control. Exp Diabetes Res 2012: 635472, 2012.

45. Buteau J, Roduit R, Susini S and Prentki M: Glucagon-like peptide-1 promotes DNA synthesis, activates phosphatidylinositol 3-kinase and increases transcription factor pancreatic and duodenal homeobox gene 1 (PDX-1) DNA binding activity in beta (INS-1)-cells. Diabetologia 42: 856-864, 1999.

46. Kanda R, Hiraike H, Wada-Hiraike O, Ichinose T, Nagasaka K, Sasajima Y, Ryo E, Fujii T, Osuga Y and Ayabe T: Expression of the glucagon-like peptide-1 receptor and its role in regulating autophagy in endometrial cancer. BMC Cancer 18: 657, 2018.

47. Zhao H, Wang L, Wei R, Xiu D, Tao M, Ke J, Liu Y, Yang J and Hong T: Activation of glucagon-like peptide-1 receptor inhibits tumourigenicity and metastasis of human pancreatic cancer cells via PI3K/Akt pathway. Diabetes Obes Metab 16: 850-860, 2014

48. Gao Q, Yu GY, Shi JY, Li LH, Zhang WJ, Wang ZC, Yang LX, Duan M, Zhao H, Wang XY, et al: Neddylation pathway is up-regulated in human intrahepatic cholangiocarcinoma and serves as a potential therapeutic target. Oncotarget 5: 7820-7832, 2014. 